\title{
A Locally Advanced Breast Cancer that Achieved pCR with Pertuzumab, Trastuzumab and Docetaxel: Case Report
}

\author{
YUKA INOUE $^{1}$, NAMI YAMASHITA ${ }^{1}$, ERIKO TOKUNAGA ${ }^{2}$, KIMIHIRO TANAKA $^{1}$, HIROKI UEO $^{1}$, \\ HIROSHI SAEKI ${ }^{1}$, EIJI OKI ${ }^{1}$, HIDETAKA YAMAMOTO $^{3}$ and YOSHIHIKO MAEHARA ${ }^{1}$ \\ ${ }^{1}$ Department of Surgery and Science, Graduate School of Medical Sciences, Kyushu University, Fukuoka, Japan; \\ ${ }^{2}$ National Hospital Organization Kyushu Cancer Center, Fukuoka, Japan; \\ ${ }^{3}$ Department of Anatomic Pathology, Pathological Sciences, \\ Graduate School of Medical Sciences, Kyushu University, Fukuoka, Japan
}

\begin{abstract}
We herein report a case of locally advanced human epidermal growth factor receptor 2 (HER2)-positive breast cancer that achieved a pathological complete response ( $p C R$ ) with pertuzumab, trastuzumab and docetaxel therapy. A 70-year-old female presented with an elastic hard mass, $5.0 \mathrm{~cm}$ in diameter with broad redness and edema of the skin in her right breast. Swollen lymph nodes were also recognized in the right axilla. The pathological diagnosis was invasive ductal carcinoma and its biological character was estrogen receptor (ER)-negative, progesterone receptor (PgR)-negative, HER2 3+ and Ki-67 index 60\%. The patient was finally diagnosed with primary unresectable, locally advanced breast cancer and started on pertuzumab, trastuzumab and docetaxel combination therapy. The tumor subsequently reduced in size and, after 4 cycles of this therapy, she underwent surgery. The histopathological examination of the postoperative specimen showed $p C R$ in both the primary tumor and axillary lymph nodes.
\end{abstract}

Approximately $20 \%$ of all breast cancers have a gene amplification or overexpression of human epidermal growth factor receptor 2 (HER2) (1), a tyrosine kinase transmembrane receptor, resulting in a more aggressive phenotype and a poor prognosis (2). However, the emergence of anti-HER2 therapies has improved the outcome of patients with HER2positive breast cancer.

Recently, new anti-HER2 therapies for HER2-positive advanced or metastatic breast cancer was approved in Japan.

Correspondence to: Yoshihiko Maehara, Department of Surgery and Science, Graduate, School of Medical Sciences, Kyushu University, 3-1-1, Maidashi, Higashi-ku, Fukuoka, Japan. Tel: +81 926425466, Fax: +81 926425482, e-mail: maehara@surg2.med.kyushu-u.ac.jp

Key Words: Pertuzumab, anti-HER2 therapy, advanced breast cancer.
One of them was pertuzumab, a recombinant humanized monoclonal antibody that targets the extracellular, dimerization domain (subdomain II) of the HER2 protein and blocks the ligand-dependent heterodimerization of HER2 with other ligand-activated HER receptors, most notably HER3 $(3,4)$. A randomized phase III trial in metastatic breast cancer, the CLEOPATRA trial, compared the efficacy and safety of pertuzumab, trastuzumab and docetaxel versus placebo, trastuzumab and docetaxel in the first-line setting in 808 patients. The median progression-free survival was significantly better in the pertuzumab group (hazard ratio $(\mathrm{HR})=0.62 ; p<0.001)$. The overall survival (OS) was reported with a median follow-up of 30.1 months for the placebo group and 29.7 months for the pertuzumab group (5). In The National Comprehensive Cancer Network (NCCN) guideline (2016 version 1.0), this regimen is recommended for HER2-positive unresectable or metastatic breast cancer as first-line chemotherapy.

We herein report a case of locally advanced HER2-positive breast cancer that achieved pathological complete response (pCR) with pertuzumab, trastuzumab and docetaxel therapy.

\section{Case Report}

The case was a 70-year-old postmenopausal female who presented with a large mass in her right breast. She had been complaining of a palpable mass in the upper outer quadrant of the right breast for three months before admission. She had undergone surgery for an abdominal aortic aneurysm a year previously.

A physical examination revealed that an elastic hard mass, $5.0 \mathrm{~cm}$ in diameter with broad redness and edema of the skin, was observed in the upper outer quadrant of the right breast. There were also swollen lymph nodes in the right axilla. The laboratory data showed elevated levels of tumor markers: carcinoma antigen 15-3 (CA15-3) $45.9 \quad \mathrm{U} / \mathrm{ml}$ and carcinoembryonic antigen (CEA) $8.2 \mathrm{ng} / \mathrm{ml}$. Mammography 
a

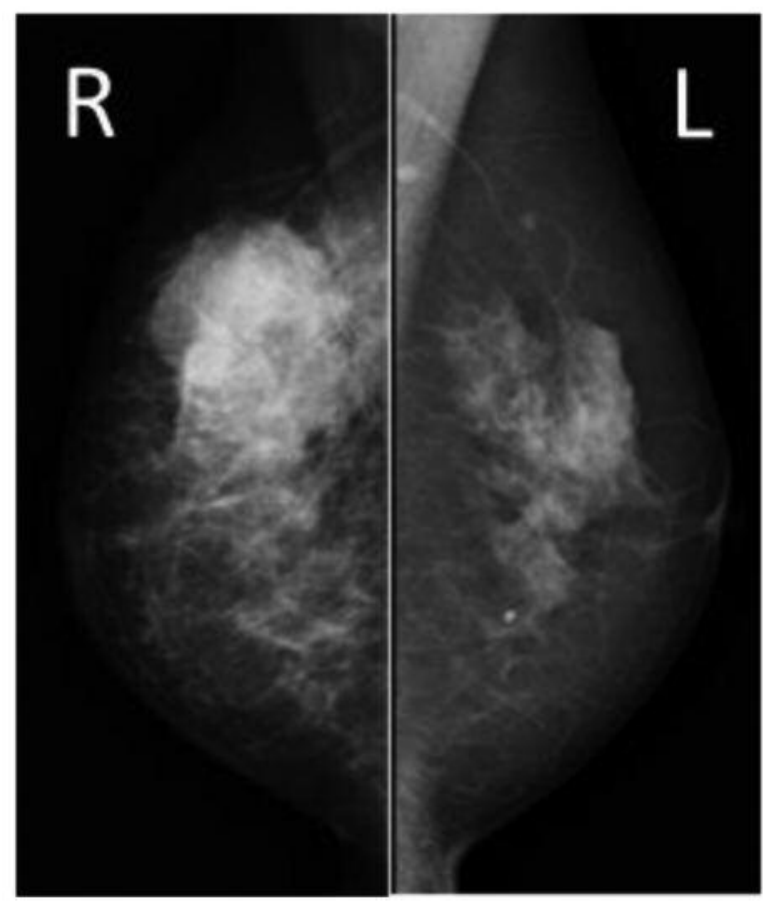

b

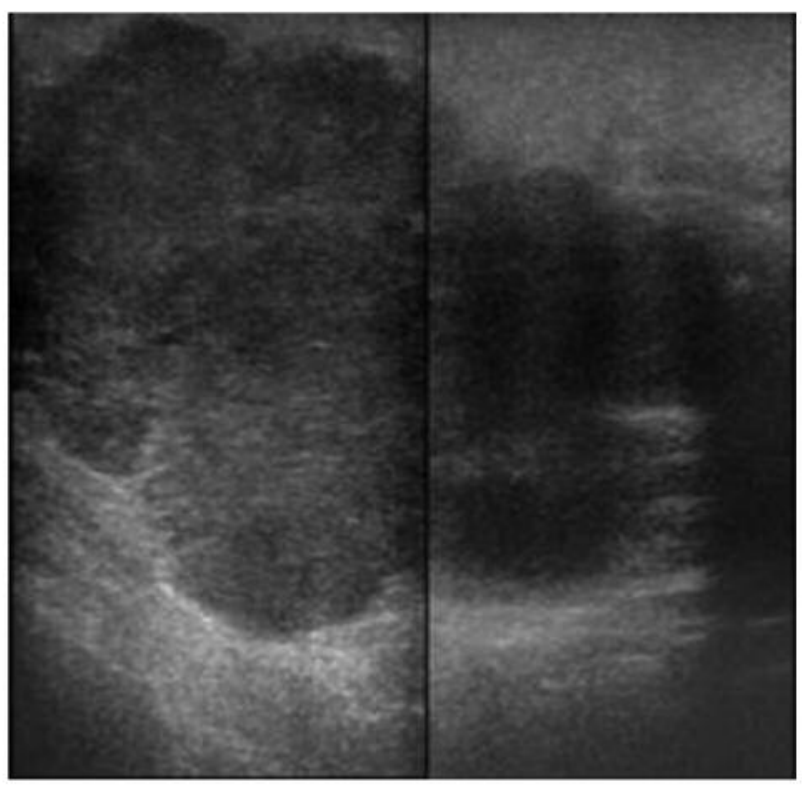

d

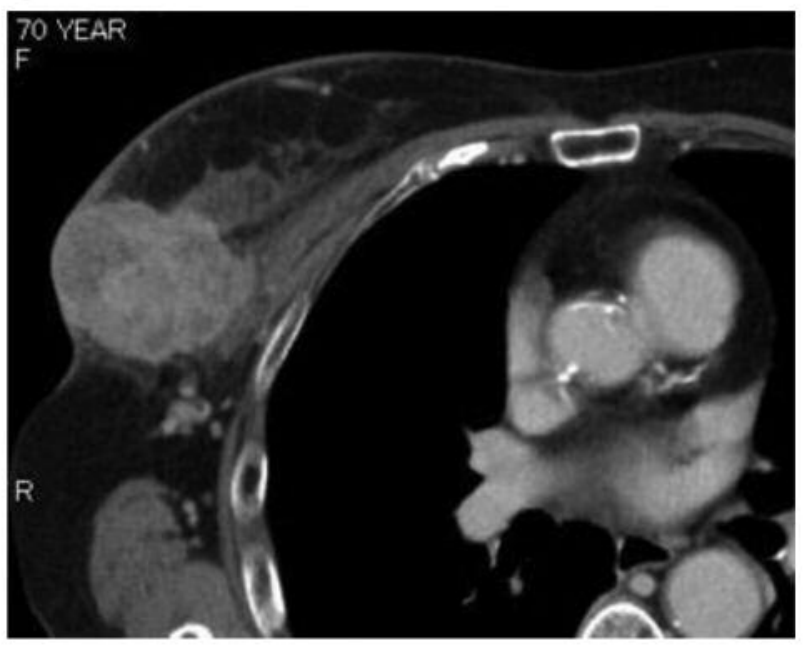

Figure 1. Imaging findings before pertuzumab, trastuzumab and docetaxel therapy. (a) Mammography showed an irregular, high-density mass. (b) Ultrasonography confirmed the presence of a 58-mm in diameter, lobulated, low-echoic area. (c) Pre-treatment magnetic resonance imaging (MRI) showed a lobulated mass and the tumor showed a rapid-washout pattern. (d) Pre-treatment computed tomography (CT) revealed that the tumor had invaded to the skin and the muscle.

revealed a $5.0 \mathrm{~cm}$ irregular mass in the right upper area in the right mediolateral oblique position (Figure 1a). Ultrasonography revealed an irregular low-echoic mass, $5.8 \mathrm{~cm}$ in diameter, in the upper outer quadrant of the right breast (Figure 1b) and swollen lymph nodes in the right axilla. Magnetic resonance imaging (MRI) revealed a $5.8 \mathrm{~cm}$ high-intensity mass in the upper outer area of the right breast, which was enhanced by gadolinium from the early phase (Figure 1c). A computed tomography (CT) scan revealed that the tumor had diffuse invasion to the skin and the pectoralis major muscle. There was no distant metastasis (Figure 1d). A core needle biopsy from the tumor indicated that the tumor was invasive ductal carcinoma (Figure 2a). Immunohistochemistry demonstrated tumor cells that were estrogen receptor (ER)-negative (Figure 2b), progesterone receptor (PgR)-negative (Figure 2c), HER2 score $3+$ (Figure 2d) and Ki-67 60\% (Figure 2e). 
a
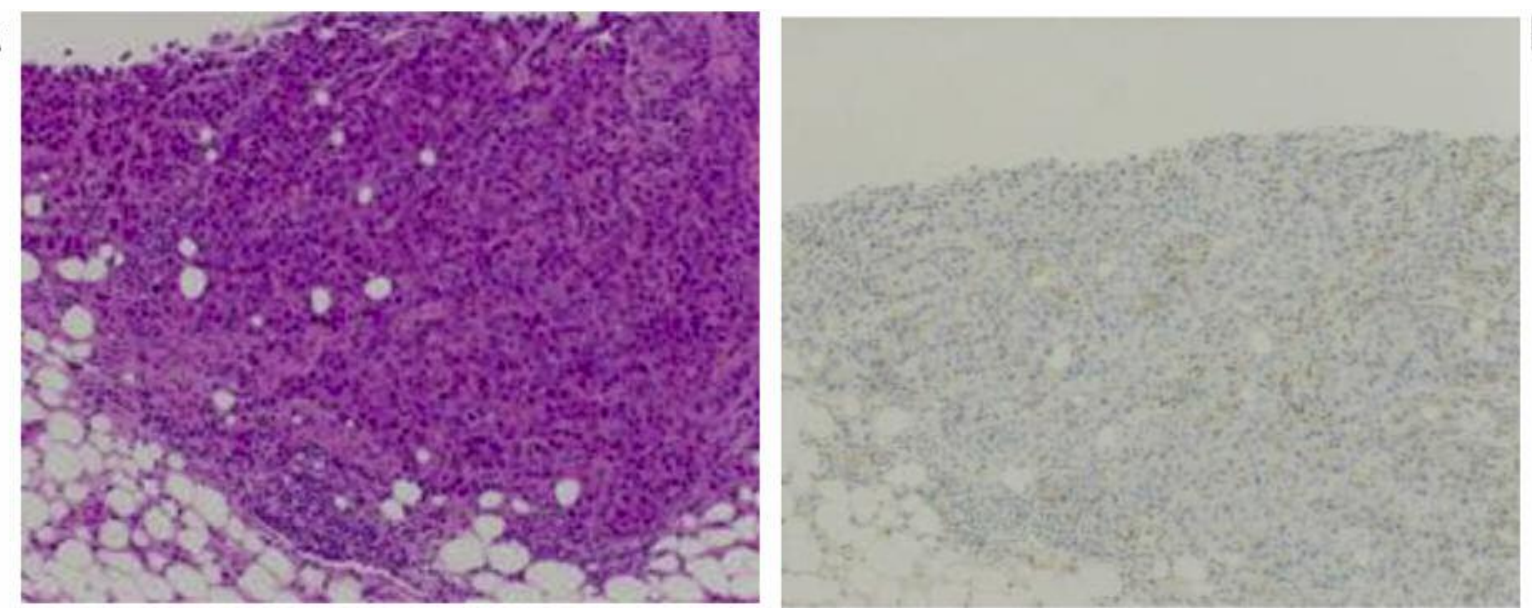

b

C
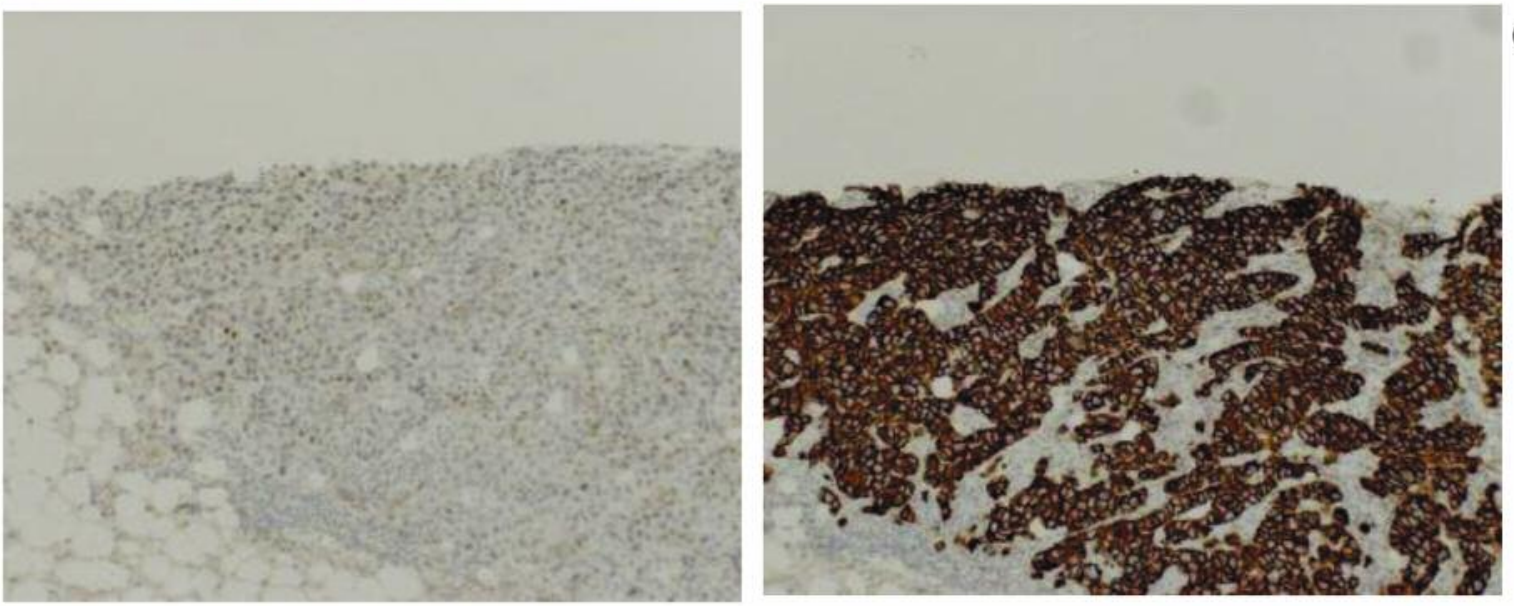

e

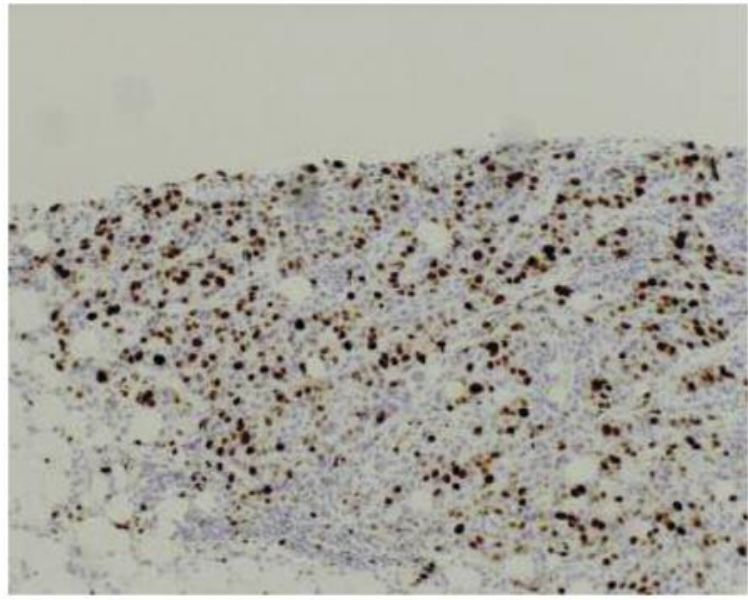

Figure 2. Pathological findings of hematoxylin and eosin (H\&E) staining and immunohistochemistry. (a) A core-needle biopsy revealed that the tumor was invasive ductal carcinoma (100x). (b) Eestrogen receptor (ER)-negative (100x). (c) Progesterone receptor (PgR)-negative (100x). (d) Human epidermal growth factor receptor 2 (HER2) score 3+ (100x). (e) Ki-67 60\% (100x).

According to the clinical and pathological findings, the patient was diagnosed with primarily unresectable HER2positive breast cancer and the clinical stage was T4bN1M0
Stage IIIB, according to Union for International Cancer Control (UICC) TNM Classification of Malignant Tumors (7th edition). Initially, the patient was treated with pertuzumab, 


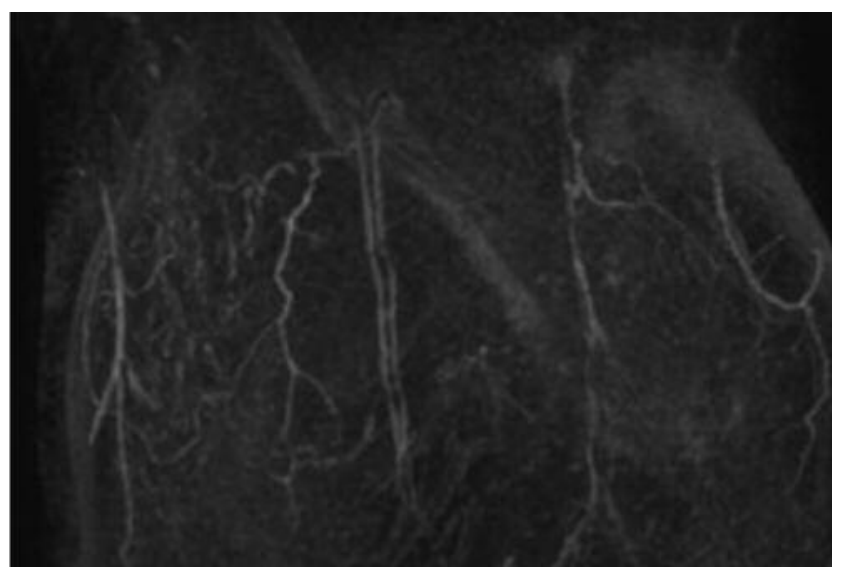

Figure 3. After 4 cycles of chemotherapy, magnetic resonance imaging (MRI) revealed that the dynamic phase did not show an enhancing mass in the right upper outer breast.

trastuzumab and docetaxel therapy. She received a loading dose of $8 \mathrm{mg} / \mathrm{kg}$ of trastuzumab, followed by a maintenance dose of $6 \mathrm{mg} / \mathrm{kg}$ every 3 weeks. Docetaxel was administered every 3 weeks at a starting dose of $55 \mathrm{mg} / \mathrm{m}^{2}$, reduced by $20 \%$, because the patient was elderly. Pertuzumab was given at a fixed loading dose of $840 \mathrm{mg}$, followed by $420 \mathrm{mg}$ every 3 weeks. After the first course, the tumor showed a rapid size reduction and became not palpable. During the patient's treatment course, she had no adverse events of grade 3 or higher (Common Terminology Criteria for Adverse Events, version 4.0). After 4 cycles of pertuzumab, trastuzumab and docetaxel, the high-intensity mass in the right upper outer breast almost disappeared on MRI (Figure 3). A CT scan revealed diminished invasion to the skin and the pectoralis major muscle. Because the tumor had greatly reduced in size, the patient underwent surgery, right partial mastectomy and axillary lymph node dissection (levels I, II). The histopathological examination of the postoperative specimen showed pCR (Figure 4). Sections of the specimen showed mammary tissue with fibrosis, aggregates of foamy macrophages and chronic inflammatory infiltrate, carrying no carcinoma. The patient is currently being treated with trastuzumab as adjuvant therapy and has had no relapse of the breast cancer.

\section{Discussion}

In the present case, the patient was initially diagnosed with unresectable, locally advanced right breast cancer. Because the tumor greatly reduced in size following pertuzumab, trastuzumab and docetaxel therapy, the patient was able to undergo operation. Furthermore, the histopathological examination of the postoperative specimen showed pCR.

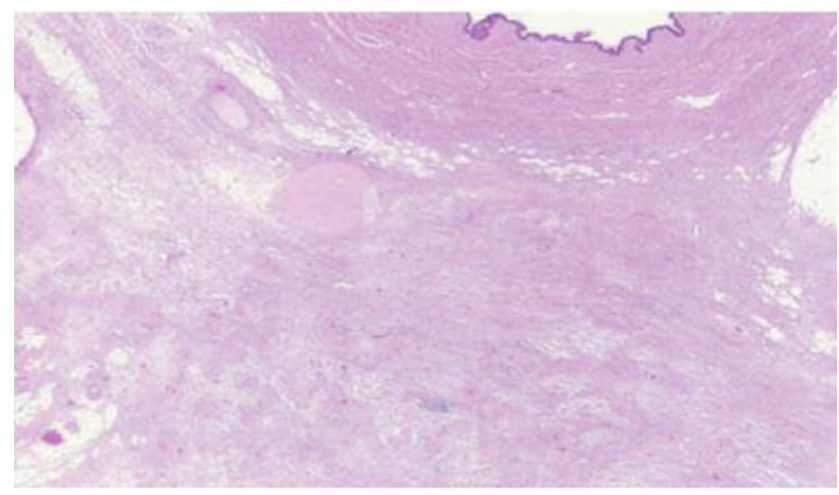

Figure 4. Pathological findings of hematoxylin and eosin $(H \& E)$ staining of the postoperative specimen. The histopathological examination of the postoperative specimen showed a pathological complete response.

Pertuzumab has not yet been approved as neoadjuvant therapy in Japan. On the other hand, according to the NCCN guideline, pertuzumab is approved in the neoadjuvant setting, with a limited use for HER2-positive breast cancer with T2 or N1, according to two randomized, open label, phase II trials in the neoadjuvant setting that demonstrated improvements in pCR (NeoSphere and TRYPHAENA) $(6,7)$.

In the neoadjuvant setting, NeoSphere enrolled 417 women with stage II or III HER2-positive breast cancer. The pCR (ypT0/is ypN0) rates were $39.3 \%$ and $21.5 \%$ in the docetaxel/trastuzumab/pertuzumab and docetaxel/trastuzumab arms, respectively, netting a $17.8 \%$ improvement in the pCR rate for patients who received pertuzumab. The pCR (ypT0/is ypN0) rates were $17.7 \%$ and $11.2 \%$ for docetaxel/pertuzumab and pertuzumab/trastuzumab, respectively (6).

The second neoadjuvant trial, TRYPHAENA, enrolled 225 women with HER2-positive breast cancer, who received six cycles of therapy with FEC/trastuzumab/pertuzumab followed by docetaxel/trastuzumab/pertuzumab versus FEC followed by docetaxel/trastuzumab/pertuzumab versus docetaxel/carboplatin/ trastuzumab/pertuzumab. The pCR rates for the three arms were all even higher than those seen in the NeoSphere trial $(61.6 \%$, $57.3 \%$ and $66.2 \%$, respectively), reflecting the inclusion of all chemotherapy in the neoadjuvant setting (7). These results suggest that neoadjuvant therapy using pertuzumab, trastuzumab and docetaxel is effective for patients with HER2positive breast cancer.

In the present case, the patient was a 70-year-old; however, she had no adverse events of grade 3 or higher following pertuzumab, trastuzumab and docetaxel therapy. According to the results from a subgroup analysis of the CLEOPATRA trial, the efficacy and safety data suggest that the combined use of pertuzumab, trastuzumab and docetaxel should not be 
limited by patient age. However, dose reductions of docetaxel below $75 \mathrm{mg} / \mathrm{m}^{2}$ occurred more often in patients 65 years of age or older than in younger patients (8). In this case, the patient was treated at a dose of $55 \mathrm{mg} / \mathrm{m}^{2}$, reduced by $20 \%$ from the initial dose. This result suggests that the dose of docetaxel is important for tolerance of pertuzumab, trastuzumab and docetaxel administration in older patients.

Recently, several groups have reported that the associations between the pCR and long-term outcomes (event-free survival (EFS) and overall survival (OS)) are different among subtypes of breast cancer. An association between pCR and better long-term outcomes is strongest in patients with HER2-positive, hormone-receptor-negative tumors (9).

\section{Conclusion}

HER2-targeted therapies are important and effective for HER2-positive breast cancer. However, further investigations are necessary for an effective use of HER2-targeted therapies. Additional biomarker analyses from ongoing studies may help us predict the efficacy of HER2-targeted therapies and select patients more adequately.

\section{Acknowledgements}

No funding was received for this study.

\section{References}

1 Wolff AC, Hammond ME, Schwartz JN, Hagerty KL, Allred DC, Cote RJ, Dowsett M, Fitzgibbons PL, Hanna WM, Langer A, McShane LM, Paik S, Pegram MD, Perez EA, Press MF, Rhodes A, Sturgeon C, Taube SE, Tubbs R, Vance GH, van de Vijver M, Wheeler TM and Hayes DF: American Society of Clinical Oncology/College of American Pathologists guideline recommendations for human epidermal growth factor receptor 2 testing in breast cancer. Arch Pathol Lab Med 131: 18-43, 2007.

2 Ross JS, Slodkowska EA, Symmans WF, Pusztai L, Ravdin PM and Hortobagyi GN: The HER-2 receptor and breast cancer: ten years of targeted anti-HER-2 therapy and personalized medicine. Oncologist 14: 320-368, 2009.

3 Lee-Hoeflich ST, Crocker L, Yao E, Pham T, Munroe X, Hoeflich KP, Sliwkowski MX and Stern HM: A central role for HER3 in HER2-amplified breast cancer: Implications for targeted therapy. Cancer Res 68: 5878-5887, 2008.
4 Arpino G, Gutierrez C, Weiss H, Rimawi M, Massarweh S, Bharwani L, De Placido S, Osborne CK and Schiff R: Treatment of human epidermal growth factor receptor 2-overexpressing breast cancer xenografts with multiagent HER-targeted therapy. J Natl Cancer Inst 99: 694-705, 2007.

5 Swain SM, Kim SB, Cortés J, Ro J, Semiglazov V, Campone M, Ciruelos E, Ferrero JM, Schneeweiss A, Knott A, Clark E, Ross $\mathrm{G}$, Benyunes MC and Baselga J: Pertuzumab, trastuzumab, and docetaxel for HER2-positive metastatic breast cancer (CLEOPATRA study): Overall survival results from a randomised, double-blind, placebo-controlled, phase 3 study. Lancet Oncol 14: 461-471, 2013.

6 Gianni L, Pienkowski T, Im YH, Roman L, Tseng LM, Liu MC, Lluch A, Staroslawska E, de la Haba-Rodriguez J, Im SA, Pedrini JL, Poirier B, Morandi P, Semiglazov V, Srimuninnimit V, Bianchi G, Szado T, Ratnayake J, Ross G and Valagussa P: Efficacy and safety of neoadjuvant pertuzumab and trastuzumab in women with locally advanced, inflammatory, or early HER2positive breast cancer (NeoSphere): A randomised multicentre, open-label, phase 2 trial. Lancet Oncol 13: 25-32, 2012.

7 Schneeweiss A, Chia S, Hickish T, Harvey V, Eniu A, Hegg R, Tausch C, Seo JH, Tsai YF, Ratnayake J, McNally V, Ross G and Cortés J: Pertuzumab plus trastuzumab in combination with standard neoadjuvant anthracycline-containing and anthracyclinefree chemotherapy regimens in patients with HER2-positive early breast cancer: A randomized phase II cardiac safety study (TRYPHAENA). Ann Oncol 24: 2278-2284, 2013.

8 Miles D, Baselga J, Amadori D, Sunpaweravong P, Semiglazov V, Knott A, Clark E, Ross G and Swain SM: Treatment of older patients with HER2-positive metastatic breast cancer with pertuzumab, trastuzumab, and docetaxel: subgroup analyses from a randomized, double-blind, placebo-controlled phase III trial (CLEOPATRA). Breast Cancer Res Treat 142: 89-99, 2013.

9 Cortazar P, Zhang L, Untch M, Mehta K, Costantino JP, Wolmark N, Bonnefoi H, Cameron D, Gianni L, Valagussa P, Swain SM, Prowell T, Loibl S, Wickerham DL, Bogaerts J, Baselga J, Perou C, Blumenthal G, Blohmer J, Mamounas EP, Bergh J, Semiglazov V, Justice R, Eidtmann H, Paik S, Piccart M, Sridhara R, Fasching PA, Slaets L, Tang S, Gerber B, Geyer CE Jr, Pazdur R, Ditsch N, Rastogi P, Eiermann W and von Minckwitz G: Pathological complete response and long-term clinical benefit in breast cancer: The CTNeoBC pooled analysis. Lancet 384: 164-172, 2014.

Received February 7, 2017

Revised March 6, 2017

Accepted March 7, 2017 